Korean J. Math. 22 (2014), No. 3, pp. 443-454

http://dx.doi.org/10.11568/kjm.2014.22.3.443

\title{
LINEARLIZATION OF GENERALIZED FIBONACCI SEQUENCES
}

\author{
Young Ho Jang And SAng Pyo Jun ${ }^{\dagger}$
}

\begin{abstract}
In this paper, we give linearization of generalized Fibonacci sequences $\left\{g_{n}\right\}$ and $\left\{q_{n}\right\}$, respectively, defined by Eq. (5) and Eq.(6) below and use this result to give the matrix form of the $n$th power of a companion matrix of $\left\{g_{n}\right\}$ and $\left\{q_{n}\right\}$, respectively. Then we re-prove the Cassini's identity for $\left\{g_{n}\right\}$ and $\left\{q_{n}\right\}$, respectively.
\end{abstract}

\section{Introduction}

Let $Q=\left(\begin{array}{ll}1 & 1 \\ 1 & 0\end{array}\right)$ be a companion matrix of the Fibonacci sequence $\left\{f_{n}\right\}$ defined by the second-order linear recurrence relation

$$
f_{0}=0, f_{1}=1, f_{n}=f_{n-1}+f_{n-2}(n \geq 2) .
$$

Then, by an inductive argument ([10], [7], [8]), the $n$th power $Q^{n}$ has the matrix form

$$
Q^{n}=\left(\begin{array}{cc}
f_{n+1} & f_{n} \\
f_{n} & f_{n-1}
\end{array}\right)(n \geq 1)
$$

Received June 28, 2014. Revised September 15, 2014. Accepted September 15, 2014.

2010 Mathematics Subject Classification: 11B39.

Key words and phrases: generalized Fibonacci sequences, Binet's formula, companion matrix, Cassini's identity.

$\dagger$ Funding for this paper was provided by Namseoul University.

(c) The Kangwon-Kyungki Mathematical Society, 2014.

This is an Open Access article distributed under the terms of the Creative commons Attribution Non-Commercial License (http://creativecommons.org/licenses/by -nc/3.0/) which permits unrestricted non-commercial use, distribution and reproduction in any medium, provided the original work is properly cited. 
This property provides an alternate proof of the Cassini's identity for $\left\{f_{n}\right\}$

$$
f_{n-1} f_{n+1}-f_{n}^{2}=(-1)^{n}(n \geq 1) .
$$

Now, let's think of the other access method in order to give the matrix form Eq.(1) of $Q^{n}$. This method give the motivation of our research. That is, our research is based on the following observation: It is well known [5] that the usual Fibonacci numbers can be expressed using Binet's formula

$$
f_{n}=\frac{1}{\sqrt{5}}\left[\left(\frac{1+\sqrt{5}}{2}\right)^{n}-\left(\frac{1-\sqrt{5}}{2}\right)^{n}\right]=\frac{\alpha^{n}-\beta^{n}}{\alpha-\beta},
$$

where $\alpha, \beta$ are the roots of the quadratic equation $x^{2}-x-1=0$ and $\alpha>\beta$. From the Binet's formula Eq.(2), we have for any integer $n \geq 1$

(3) $f_{n}-\beta f_{n-1}=\frac{\alpha^{n}-\beta^{n}}{\alpha-\beta}-\frac{\beta\left(\alpha^{n-1}-\beta^{n-1}\right)}{\alpha-\beta}=\frac{\alpha^{n-1}(\alpha-\beta)}{\alpha-\beta}=\alpha^{n-1}$.

Multipling Eq.(3) by $\alpha$, using $\alpha \beta=-1$, and if we change $\alpha$ and $\beta$ role above process, we obtain the linearization of $\left\{f_{n}\right\}$

$$
\text { Linearization of }\left\{f_{n}\right\}:\left\{\begin{array}{l}
\alpha^{n}=f_{n} \alpha+f_{n-1} \\
\beta^{n}=f_{n} \beta+f_{n-1}
\end{array}\right.
$$

In Eq.(4), if we change $\alpha, \beta$ into the companion matrix $Q$ and change $f_{n-1}$ into the matrix $f_{n-1} I$, where $I$ is the $2 \times 2$ identity matrix, then we obtain the matrix form Eq.(1) of $Q^{n}$

$$
Q^{n}=f_{n} Q+f_{n-1} I\left(=\left(\begin{array}{cc}
f_{n+1} & f_{n} \\
f_{n} & f_{n-1}
\end{array}\right)\right) .
$$

The Fibonacci sequence has been generalized in many ways, for example, by changing the recurrence relation while preserving the initial terms, by altering the initial terms but maintaining the recurrence relation, by combining of these two techniques, and so on (for more details see $[2,3,4,7,11])$.

In this paper, we consider two types of generalized Fibonacci sequences which are basically different. One is the sequence $\left\{g_{n}\right\}$ defined by Gupta et al. [4] depending on four positive integer parameters $g_{0}, g_{1}, a$ and $b$ used in the secon-order linear recurrence relation:

$$
g_{n}=a g_{n-1}+b g_{n-2}(n \geq 2)
$$


Another is the sequence $\left\{q_{n}\right\}$ defined by Edson et al. [2] depending on two positive integer parameters $a$ and $b$ used in the secon-order nonlinear recurrence relation:

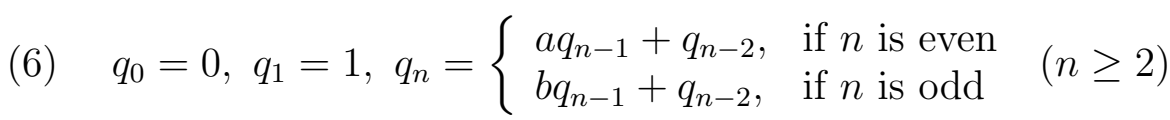

In this paper, as mentioned above, we provide linearlization of $\left\{g_{n}\right\}$ and $\left\{q_{n}\right\}$, respectively, and use this result to give the matrix form of the $n$th power of a companion matrix of $\left\{g_{n}\right\}$ and $\left\{q_{n}\right\}$, repectively. Then we re-prove the Cassini's identity for $\left\{g_{n}\right\}$ and $\left\{q_{n}\right\}$, respectively.

\section{Linearlization of the generalized Fibonacci sequences $\left\{g_{n}\right\}$}

Many number theory texts(see for example, Niven and Zuckermann [9]) prove that the analogous Binet's formula for the generalized Fibonacci sequence $\left\{g_{n}\right\}$ defined by Eq.(5) is

$$
(\alpha-\beta) g_{n}=g_{1}\left(\alpha^{n}-\beta^{n}\right)+g_{0}\left(\alpha \beta^{n}-\beta \alpha^{n}\right),
$$

where $\alpha, \beta$ are the roots of the quadratic equation $x^{2}-a x-b=0$ provided $a^{2}+4 b \neq 0$.

In [12], using an inductive argument, authors give the matrix form of the $n$th power of a companion matrix $M=\left(\begin{array}{ll}a & b \\ 1 & 0\end{array}\right)$ of $\left\{g_{n}\right\}$

$$
M^{n}\left(\begin{array}{cc}
g_{2} & g_{1} \\
g_{1} & g_{0}
\end{array}\right)=\left(\begin{array}{cc}
g_{n+2} & g_{n+1} \\
g_{n+1} & g_{n}
\end{array}\right) .
$$

And then give the Cassini's identity for $\left\{g_{n}\right\}$ by taking determinant both sides of the matrix form Eq.(8)

$$
g_{n} g_{n+2}-g_{n+1}^{2}=(-b)^{n}\left(g_{0} g_{2}-g_{1}^{2}\right) .
$$

In this subsection, we give the linealization of $\left\{g_{n}\right\}$ and then use this result to obtain the matrix form Eq.(8).

Theorem 2.1. Let $\left\{g_{n}\right\}, \alpha$ and $\beta$ be as above. Then we have for all integer $n \geq 1$

$$
\text { Linearization of }\left\{g_{n}\right\}:\left\{\begin{array}{l}
\alpha^{n}\left(g_{1} \alpha+b g_{0}\right)=g_{n+1} \alpha+b g_{n} \\
\beta^{n}\left(g_{1} \beta+b g_{0}\right)=g_{n+1} \beta+b g_{n}
\end{array}\right.
$$


Proof. Using the Binet's formula Eq.(7), we have

$$
\begin{aligned}
& (\alpha-\beta) g_{n+1}-\beta(\alpha-\beta) g_{n} \\
= & g_{1}\left(\alpha^{n+1}-\beta^{n+1}\right)+g_{0}\left(\alpha \beta^{n+1}-\beta \alpha^{n+1}\right) \\
& \quad-g_{1} \beta\left(\alpha^{n}-\beta^{n}\right)-g_{0}\left(\alpha \beta^{n+1}-\beta^{2} \alpha^{n}\right) \\
= & g_{1} \alpha^{n}(\alpha-\beta)-g_{0} \beta \alpha^{n}(\alpha-\beta) .
\end{aligned}
$$

Since $\alpha \neq \beta$, we get

$$
g_{n+1}-\beta g_{n}=g_{1} \alpha^{n}-g_{0} \beta \alpha^{n} .
$$

Multiplying Eq.(11) by $\alpha$ and using $\alpha \beta=-b$, we have

$$
\alpha g_{n+1}+b g_{n}=g_{1} \alpha^{n+1}+b g_{0} \alpha^{n}=\alpha^{n}\left(g_{1} \alpha+b g_{0}\right)
$$

If we change $\alpha$ and $\beta$ role above process, we obtain the desired result Eq.(10).

We can re-prove equations Eq.(8) and Eq.(9) by using the linearlization Eq.(10) of $\left\{g_{n}\right\}$.

Corollary 2.2. Let $M=\left(\begin{array}{ll}a & b \\ 1 & 0\end{array}\right)$ be a companion matrix of $\left\{g_{n}\right\}$. Then the matrix form of the $n$th power $M^{n}$ is given by Eq.(8) and the Cassini's identity for $\left\{g_{n}\right\}$ is given by Eq.(9).

Proof. In Eq.(10), if we change $\alpha, \beta$ into the matrix $M$ and change $b g_{n}$ into the matrix $b g_{n} I$, then we have

$$
M^{n}\left(g_{1} M+b g_{0} I\right)=g_{n+1} M+b g_{n} I
$$

In fact, Eq.(12) holds for the following reason: Since

$$
M\left(\begin{array}{c}
g_{n} \\
g_{n-1}
\end{array}\right)=\left(\begin{array}{c}
g_{n+1} \\
g_{n}
\end{array}\right) \text { and } M^{n}\left(\begin{array}{c}
g_{1} \\
g_{0}
\end{array}\right)=\left(\begin{array}{c}
g_{n+1} \\
g_{n}
\end{array}\right)
$$


we have

$$
\begin{aligned}
& M^{n}\left(g_{1} M+b g_{0} I\right)\left(\begin{array}{c}
g_{1} \\
g_{0}
\end{array}\right) \\
= & g_{1} M^{n+1}\left(\begin{array}{c}
g_{1} \\
g_{0}
\end{array}\right)+b g_{0} M^{n}\left(\begin{array}{c}
g_{1} \\
g_{0}
\end{array}\right)=g_{1}\left(\begin{array}{c}
g_{n+2} \\
g_{n+1}
\end{array}\right)+b g_{0}\left(\begin{array}{c}
g_{n+1} \\
g_{n}
\end{array}\right) \\
= & g_{1}\left(\begin{array}{c}
a g_{n+1}+b g_{n} \\
g_{n+1}
\end{array}\right)+b g_{0}\left(\begin{array}{c}
g_{n+1} \\
g_{n}
\end{array}\right)=\left(\begin{array}{c}
b g_{1} g_{n}+\left(a g_{1}+b g_{0}\right) g_{n+1} \\
b g_{0} g_{n}+g_{1} g_{n+1}
\end{array}\right) \\
= & \left(\begin{array}{c}
b g_{1} g_{n}+g_{2} g_{n+1} \\
b g_{0} g_{n}+g_{1} g_{n+1}
\end{array}\right)=g_{n+1}\left(\begin{array}{c}
g_{2} \\
g_{1}
\end{array}\right)+b g_{n}\left(\begin{array}{c}
g_{1} \\
g_{0}
\end{array}\right) \\
= & g_{n+1} M\left(\begin{array}{c}
g_{1} \\
g_{0}
\end{array}\right)+b g_{n}\left(\begin{array}{c}
g_{1} \\
g_{0}
\end{array}\right)=\left(g_{n+1} M+b g_{n} I\right)\left(\begin{array}{c}
g_{1} \\
g_{0}
\end{array}\right) .
\end{aligned}
$$

Thus from Eq.(12) we have

$$
\begin{aligned}
M^{n}\left(g_{1} M+b g_{0} I\right)=M^{n}\left(\begin{array}{cc}
a g_{1}+b g_{0} & b g_{1} \\
g_{1} & b g_{0}
\end{array}\right) & =M^{n}\left(\begin{array}{ll}
g_{2} & b g_{1} \\
g_{1} & b g_{0}
\end{array}\right) \\
& =M^{n}\left(\begin{array}{ll}
g_{2} & g_{1} \\
g_{1} & g_{0}
\end{array}\right)\left(\begin{array}{ll}
1 & 0 \\
0 & b
\end{array}\right), \\
g_{n+1} M+b g_{n} I=\left(\begin{array}{cc}
a g_{n+1}+b g_{n} & b g_{n+1} \\
g_{n+1} & b g_{n}
\end{array}\right) & =\left(\begin{array}{cc}
g_{n+2} & b g_{n+1} \\
g_{n+1} & b g_{n}
\end{array}\right) \\
& =\left(\begin{array}{cc}
g_{n+2} & g_{n+1} \\
g_{n+1} & g_{n}
\end{array}\right)\left(\begin{array}{ll}
1 & 0 \\
0 & b
\end{array}\right) .
\end{aligned}
$$

Since the matrix $\left(\begin{array}{ll}1 & 0 \\ 0 & b\end{array}\right)$ is invertible, we obtain the desired result Eq.(8) and by taking determinant both sides of the matrix form Eq.(8) we obtain the desired result Eq.(9).

\section{Linearlization of the generalized Fibonacci sequences $\left\{q_{n}\right\}$}

Edson et al. [2] give the generating function for the generalized Fibonacci sequence $\left\{q_{n}\right\}$ defined by Eq. (6)

$$
F(x)=\frac{x\left(1+a x-x^{2}\right)}{1-(a b+2) x^{2}+x^{4}}
$$


and then give the extended Binet's formula by using the generating function $F(x)$

$$
q_{n}=\left(\frac{a^{1-\xi(n)}}{(a b)^{\frac{n-\xi(n)}{2}}}\right) \frac{\alpha^{n}-\beta^{n}}{\alpha-\beta},
$$

where $\alpha, \beta$ are the roots of the quadratic equation $x^{2}-a b x-a b=0$ provided $a^{2} b^{2}+4 a b \neq 0$ and

$$
\xi(n)= \begin{cases}0 & \text { if } n \text { is even } \\ 1 & \text { if } n \text { is odd }\end{cases}
$$

is the parity function. Also, using the extended Binet's formula Eq.(13), give the Cassini's identity:

$$
a^{1-\xi(n)} b^{\xi(n)} q_{n-1} q_{n+1}-a^{\xi(n)} b^{1-\xi(n)} q_{n}^{2}=a(-1)^{n} .
$$

In this subsection, we give the linealization of $\left\{q_{n}\right\}$ and then use this result to obtain the matrix form of the $n$th power of a companion matrix of $\left\{q_{n}\right\}$.

Theorem 3.1. Let $\left\{q_{n}\right\}, \alpha, \beta$ and $\xi(n)$ be as above. Then we have for all integer $n \geq 1$

Linearization of $\left\{q_{n}\right\}:\left\{\begin{array}{l}\alpha^{n}=a^{-1} a^{\frac{n+\xi(n)}{2}} b^{\frac{n-\xi(n)}{2}} q_{n} \alpha+a^{\frac{n-\xi(n)}{2}} b^{\frac{n+\xi(n)}{2}} q_{n-1}, \\ \beta^{n}=a^{-1} a^{\frac{n+\xi(n)}{2}} b^{\frac{n-\xi(n)}{2}} q_{n} \beta+a^{\frac{n-\xi(n)}{2}} b^{\frac{n+\xi(n)}{2}} q_{n-1} .\end{array}\right.$

Proof. Since Eq.(16) holds for $n=1$, let $n \geq 2$. Using the extended Binet's formula Eq.(13), we have

$$
\begin{aligned}
& q_{n}-\frac{\beta^{2}}{a b} q_{n-2} \\
= & \left(\frac{a^{1-\xi(n)}}{(a b)^{\frac{n-\xi(n)}{2}}}\right) \frac{\alpha^{n}-\beta^{n}}{\alpha-\beta}-\frac{\beta^{2}}{a b}\left(\frac{a^{1-\xi(n-2)}}{(a b)^{\frac{n-2-\xi(n-2)}{2}}}\right) \frac{\alpha^{n-2}-\beta^{n-2}}{\alpha-\beta} \\
= & \frac{\alpha^{n-2}}{\alpha-\beta}\left(\frac{a^{1-\xi(n)}}{(a b)^{\frac{n-\xi(n)}{2}}} \alpha^{2}-\frac{a^{1-\xi(n-2)}}{(a b)^{\frac{n-\xi(n-2)}{2}}} \beta^{2}\right) \\
& \quad-\frac{\beta^{n}}{\alpha-\beta}\left(\frac{a^{1-\xi(n)}}{(a b)^{\frac{n-\xi(n)}{2}}}-\frac{a^{1-\xi(n-2)}}{(a b)^{\frac{n-\xi(n-2)}{2}}}\right) .
\end{aligned}
$$


Since $\xi(n)=\xi(n-2)$ and $\alpha+\beta=a b$, we get

$$
q_{n}-\frac{\beta^{2}}{a b} q_{n-2}=\frac{a^{1-\xi(n)}}{(a b)^{\frac{n-\xi(n)}{2}-1}} \alpha^{n-2} .
$$

Multiplying Eq.(17) by $\frac{\alpha^{2}}{a b}=\alpha+1$ and using $\alpha \beta=-a b$, we have

$$
q_{n} \alpha+\left(q_{n}-q_{n-2}\right)=\frac{a^{1-\xi(n)}}{(a b)^{\frac{n-\xi(n)}{2}}} \alpha^{n} .
$$

From the definitions Eq.(6) and Eq.(14), we have $q_{n}-q_{n-2}$ $=a^{1-\xi(n)} b^{\xi(n)} q_{n-1}$. Thus we have

$$
q_{n} \alpha+a^{1-\xi(n)} b^{\xi(n)} q_{n-1}=\frac{a^{1-\xi(n)}}{(a b)^{\frac{n-\xi(n)}{2}}} \alpha^{n} .
$$

Also, if we change $\alpha$ and $\beta$ role above process, we obtain the desired result Eq.(16).

Remark. For some positive integer $k$, if $a=b=k$, then $\left\{q_{n}\right\}$ is the $k$-Fibonacci sequence $\left\{f_{k, n}\right\}$ (for more details see [1]). In this case, let $Q=\left(\begin{array}{cc}k & 1 \\ 1 & 0\end{array}\right)$ be a companion matrix of $\left\{f_{k, n}\right\}$ and

$$
\phi=\frac{1}{2}\left(k+\sqrt{k^{2}+4}\right), \varphi=\frac{1}{2}\left(k-\sqrt{k^{2}+4}\right)
$$

be the roots of the quadratic equation $x^{2}-k x-1=0$ provided $k^{2}+4 \neq 0$. Then

$$
\begin{gathered}
\alpha=\frac{1}{2}\left(k^{2}+\sqrt{k^{4}+4 k^{2}}\right)=k \phi, \beta=\frac{1}{2}\left(k^{2}-\sqrt{k^{4}+4 k^{2}}\right)=k \varphi, \\
a^{-1} a^{\frac{n+\xi(n)}{2}} b^{\frac{n-\xi(n)}{2}}=k^{n-1}, a^{\frac{n-\xi(n)}{2}} b^{\frac{n+\xi(n)}{2}}=k^{n} .
\end{gathered}
$$

Thus we have

$$
\begin{aligned}
\text { Eq.(16) } & \Leftrightarrow\left\{\begin{array}{l}
(k \phi)^{n}=k^{n-1} f_{k . n} k \phi+k^{n} f_{k, n-1}, \\
(k \varphi)^{n}=k^{n-1} f_{k . n} k \varphi+k^{n} f_{k, n-1} .
\end{array}\right. \\
& \Leftrightarrow\left\{\begin{array}{l}
\phi^{n}=f_{k, n} \phi+f_{k, n-1}, \\
\varphi^{n}=f_{k, n} \varphi+f_{k, n-1} .
\end{array}\right.
\end{aligned}
$$

and if we change $\phi, \varphi$ into the matrix $Q$ and change $f_{k, n-1}$ into the matrix $f_{k, n-1} I$, then the matrix form of the $n$th power $Q^{n}$ is given by

$$
Q^{n}=f_{k, n} Q+f_{k, n-1} I=\left(\begin{array}{cc}
f_{k, n+1} & f_{k, n} \\
f_{k, n} & f_{k, n-1}
\end{array}\right) \quad \text { (see [6], page 2) }
$$


and we obtain the Cassini's identity for $\left\{f_{k, n}\right\}$

$$
f_{k, n-1} f_{k, n+1}-f_{k, n}^{2}=(-1)^{n} \quad \text { (see [1], Proposition 3). }
$$

LemMa 3.2. Let $M=\left(\begin{array}{cc}a b & b \\ a & 0\end{array}\right)$ be a companion matrix of the generalized Fibonacci sequence $\left\{q_{n}\right\}$ defined by Eq.(6). Then we have for all integer $n \geq 1$,

$$
M^{2 n-1}\left(\begin{array}{c}
q_{1} \\
q_{0}
\end{array}\right)=(a b)^{n-1}\left(\begin{array}{c}
b q_{2 n} \\
a q_{2 n-1}
\end{array}\right)
$$

and

$$
M^{2 n}\left(\begin{array}{c}
q_{1} \\
q_{0}
\end{array}\right)=(a b)^{n}\left(\begin{array}{c}
q_{2 n+1} \\
q_{2 n}
\end{array}\right) .
$$

Proof. We will use the induction method on $n$. If $n=1$, then

LHS of Eq. $(18)=M\left(\begin{array}{c}q_{1} \\ q_{0}\end{array}\right)=\left(\begin{array}{cc}a b & b \\ a & 0\end{array}\right)\left(\begin{array}{c}q_{1} \\ q_{0}\end{array}\right)=\left(\begin{array}{c}b\left(a q_{1}+q_{0}\right) \\ a q_{1}\end{array}\right)=\left(\begin{array}{c}b q_{2} \\ a q_{1}\end{array}\right)$

$$
=\text { RHS of Eq.(18). }
$$

We suppose that Eq.(18) holds for $n=2,3, \cdots, m$, i.e.,

$$
M^{2 n-1}\left(\begin{array}{c}
q_{1} \\
q_{0}
\end{array}\right)=(a b)^{n-1}\left(\begin{array}{c}
b q_{2 n} \\
a q_{2 n-1}
\end{array}\right) .
$$

Now, we show that Eq.(18) holds for $n=m+1$. By assumption, we have

$$
\begin{aligned}
M^{2 m+1}\left(\begin{array}{c}
q_{1} \\
q_{0}
\end{array}\right) & =M^{2}\left\{M^{2 m-1}\left(\begin{array}{l}
q_{1} \\
q_{0}
\end{array}\right)\right\}=(a b)^{m-1} M^{2}\left(\begin{array}{c}
b q_{2 m} \\
a q_{2 m-1}
\end{array}\right) \\
& =(a b)^{m-1}\left(\begin{array}{cc}
a b & b \\
a & 0
\end{array}\right)\left(\begin{array}{cc}
a b & b \\
a & 0
\end{array}\right)\left(\begin{array}{c}
b q_{2 m} \\
a q_{2 m-1}
\end{array}\right) \\
& =(a b)^{m-1}\left(\begin{array}{cc}
a b & b \\
a & 0
\end{array}\right)\left(\begin{array}{c}
a b^{2} q_{2 m}+a b q_{2 m-1} \\
a b q_{2 m}
\end{array}\right) \\
& =(a b)^{m}\left(\begin{array}{cc}
a b & b \\
a & 0
\end{array}\right)\left(\begin{array}{c}
b q_{2 m}+q_{2 m-1} \\
q_{2 m}
\end{array}\right) \\
& =(a b)^{m}\left(\begin{array}{cc}
a b & b \\
a & 0
\end{array}\right)\left(\begin{array}{c}
q_{2 m+1} \\
q_{2 m}
\end{array}\right) \\
& =(a b)^{m}\left(\begin{array}{cc}
b\left(a q_{2 m+1}+q_{2 m}\right. \\
a q_{2 m+1}
\end{array}\right)
\end{aligned}
$$




$$
=(a b)^{m}\left(\begin{array}{c}
b q_{2 m+2} \\
a q_{2 m+1}
\end{array}\right) .
$$

Next, using Eq.(18) we obtain Eq.(19) as follows:

$$
\begin{aligned}
M^{2 n}\left(\begin{array}{l}
q_{1} \\
q_{0}
\end{array}\right) & =M\left\{M^{2 n-1}\left(\begin{array}{c}
q_{1} \\
q_{0}
\end{array}\right)\right\}=M\left\{(a b)^{n-1}\left(\begin{array}{c}
b q_{2 n} \\
a q_{2 n-1}
\end{array}\right)\right\} \\
& =(a b)^{n-1}\left(\begin{array}{cc}
a b & b \\
a & 0
\end{array}\right)\left(\begin{array}{c}
b q_{2 n} \\
a q_{2 n-1}
\end{array}\right) \\
& =(a b)^{n-1}\left(\begin{array}{c}
a b^{2} q_{2 n}+a b q_{2 n-1} \\
a b q_{2 n}
\end{array}\right) \\
& =(a b)^{n}\left(\begin{array}{c}
b q_{2 n}+q_{2 n-1} \\
q_{2 n}
\end{array}\right) \\
& =(a b)^{n}\left(\begin{array}{c}
q_{2 n+1} \\
q_{2 n}
\end{array}\right) .
\end{aligned}
$$

Theorem 3.3. Let $M=\left(\begin{array}{cc}a b & b \\ a & 0\end{array}\right)$ be a companion matrix of the generalized Fibonacci sequence $\left\{q_{n}\right\}$ defined by Eq.(6). For all integer $n \geq 1$, the matrix form of the $n$th power $M^{n}$ is given by

$$
\left\{\begin{array}{l}
M^{2 n-1}=(a b)^{n-1}\left(\begin{array}{cc}
b q_{2 n} & b q_{2 n-1} \\
a q_{2 n-1} & b q_{2 n-2}
\end{array}\right), \\
M^{2 n}=(a b)^{n-1} b\left(\begin{array}{cc}
a q_{2 n+1} & b q_{2 n} \\
a q_{2 n} & a q_{2 n-1}
\end{array}\right) .
\end{array}\right.
$$

Proof. From Eq.(16) we have

$$
\begin{gathered}
\left\{\begin{array}{l}
\alpha^{2 n-1}=(a b)^{n-1}\left(q_{2 n-1} \alpha+b q_{2 n-2}\right), \\
\beta^{2 n-1}=(a b)^{n-1}\left(q_{2 n-1} \beta+b q_{2 n-2}\right),
\end{array}\right. \\
\text { and }\left\{\begin{array}{l}
\alpha^{2 n}=(a b)^{n-1} b\left(q_{2 n} \alpha+a q_{2 n-1}\right), \\
\beta^{2 n}=(a b)^{n-1} b\left(q_{2 n} \beta+a q_{2 n-1}\right) .
\end{array}\right.
\end{gathered}
$$

In Eq.(21), if we change $\alpha, \beta$ into the matrix $M$ and change $b q_{2 n-2}, a q_{2 n-1}$ into the matrix $b q_{2 n-2} I, a q_{2 n-1} I$, then we have

$$
\left\{\begin{array}{l}
M^{2 n-1}=(a b)^{n-1}\left(q_{2 n-1} M+b q_{2 n-2} I\right), \\
M^{2 n}=(a b)^{n-1} b\left(q_{2 n} M+a q_{2 n-1} I\right) .
\end{array}\right.
$$


In fact, Eq.(22) holds for the following reason: using Eq.(18) and Eq.(19) in Lemma 3.2,

$$
M^{2 n-1}\left(\begin{array}{c}
q_{1} \\
q_{0}
\end{array}\right)=(a b)^{n-1}\left(\begin{array}{c}
b q_{2 n} \\
a q_{2 n-1}
\end{array}\right)
$$

and

$$
\begin{aligned}
& (a b)^{n-1}\left(q_{2 n-1} M+b q_{2 n-2} I\right)\left(\begin{array}{l}
q_{1} \\
q_{0}
\end{array}\right) \\
= & (a b)^{n-1}\left(\begin{array}{cc}
a b q_{2 n-1}+b q_{2 n-2} & b q_{2 n-1} \\
a q_{2 n-1} & b q_{2 n-2}
\end{array}\right)\left(\begin{array}{l}
q_{1} \\
q_{0}
\end{array}\right) \\
= & (a b)^{n-1}\left(\begin{array}{c}
a b q_{2 n-1}+b q_{2 n-2} \\
a q_{2 n-1}
\end{array}\right) \\
= & (a b)^{n-1}\left(\begin{array}{c}
b\left(a q_{2 n-1}+q_{2 n-2}\right) \\
a q_{2 n-1}
\end{array}\right) \\
= & (a b)^{n-1}\left(\begin{array}{c}
b q_{2 n} \\
a q_{2 n-1}
\end{array}\right) .
\end{aligned}
$$

Similarly,

$$
M^{2 n}\left(\begin{array}{l}
q_{1} \\
q_{0}
\end{array}\right)=(a b)^{n}\left(\begin{array}{c}
q_{2 n+1} \\
q_{2 n}
\end{array}\right)
$$

and

$$
\begin{aligned}
& (a b)^{n-1} b\left(q_{2 n} M+a q_{2 n-1} I\right)\left(\begin{array}{l}
q_{1} \\
q_{0}
\end{array}\right) \\
= & (a b)^{n-1} b\left(\begin{array}{cc}
a b q_{2 n}+a q_{2 n-1} & b q_{2 n} \\
a q_{2 n} & a q_{2 n-1}
\end{array}\right)\left(\begin{array}{l}
q_{1} \\
q_{0}
\end{array}\right) \\
= & (a b)^{n-1} b\left(\begin{array}{c}
a b q_{2 n}+a q_{2 n-2} \\
a q_{2 n}
\end{array}\right) \\
= & (a b)^{n-1} b\left(\begin{array}{c}
a\left(b q_{2 n}+q_{2 n-1}\right. \\
a q_{2 n}
\end{array}\right) \\
= & (a b)^{n-1} b\left(\begin{array}{c}
a q_{2 n+1} \\
a q_{2 n}
\end{array}\right) \\
= & (a b)^{n}\left(\begin{array}{c}
q_{2 n+1} \\
q_{2 n}
\end{array}\right) .
\end{aligned}
$$


Thus from Eq.(22) we obtain the desired result Eq.(20) as follows:

$$
\begin{aligned}
M^{2 n-1} & =(a b)^{n-1}\left(q_{2 n-1} M+b q_{2 n-2} I\right) \\
& =(a b)^{n-1}\left(q_{2 n-1}\left(\begin{array}{cc}
a b & b \\
a & 0
\end{array}\right)+b q_{2 n-2}\left(\begin{array}{cc}
1 & 0 \\
0 & 1
\end{array}\right)\right) \\
& =(a b)^{n-1}\left(\begin{array}{cc}
b\left(a q_{2 n-1}+q_{2 n-2}\right) & b q_{2 n-1} \\
a q_{2 n-1} & b q_{2 n-2}
\end{array}\right) \\
& =(a b)^{n-1}\left(\begin{array}{cc}
b q_{2 n} & b q_{2 n-1} \\
a q_{2 n-1} & b q_{2 n-2}
\end{array}\right)
\end{aligned}
$$

and

$$
\begin{aligned}
M^{2 n} & =(a b)^{n-1} b\left(q_{2 n} M+a q_{2 n-1} I\right) \\
& =(a b)^{n-1} b\left(q_{2 n}\left(\begin{array}{cc}
a b & b \\
a & 0
\end{array}\right)+a q_{2 n-1}\left(\begin{array}{cc}
1 & 0 \\
0 & 1
\end{array}\right)\right) \\
& =(a b)^{n-1} b\left(\begin{array}{cc}
a\left(b q_{2 n}+q_{2 n-1}\right) & b q_{2 n} \\
a q_{2 n} & a q_{2 n-1}
\end{array}\right) \\
& =(a b)^{n-1} b\left(\begin{array}{cc}
a q_{2 n+1} & b q_{2 n} \\
a q_{2 n} & a q_{2 n-1}
\end{array}\right) .
\end{aligned}
$$

Remark. By taking determinant both sides of the matrix form Eq.(20) in Theorem 3.3, we have

$\left|\left(\begin{array}{cc}a b & b \\ a & 0\end{array}\right)^{2 n-1}\right|=\left|(a b)^{n-1}\left(\begin{array}{cc}b q_{2 n} & b q_{2 n-1} \\ a q_{2 n-1} & b q_{2 n-2}\end{array}\right)\right| \Leftrightarrow-a=b q_{2 n-2} q_{2 n}-a q_{2 n-1}^{2}$

and

$$
\left|\left(\begin{array}{cc}
a b & b \\
a & 0
\end{array}\right)^{2 n}\right|=\left|(a b)^{n-1} b\left(\begin{array}{cc}
a q_{2 n+1} & b q_{2 n} \\
a q_{2 n} & a q_{2 n-1}
\end{array}\right)\right| \Leftrightarrow a=a q_{2 n-1} q_{2 n+1}-b q_{2 n}^{2},
$$

that is, using the parity function $\xi(n)$ defined by Eq.(14), we obtain the Cassini's identity Eq.(15) for $\left\{q_{n}\right\}$

$$
a^{1-\xi(n)} b^{\xi(n)} q_{n-1} q_{n+1}-a^{\xi(n)} b^{1-\xi(n)} q_{n}^{2}=a(-1)^{n} .
$$

\section{References}

[1] P. Catarino, On some identities for $k$-Fibonacci sequence, Int. J. Contemp. Math. Sciences, 9 (2014), 37-42. 
[2] M. Edson and O. Yayenie, A new generalization of Fibonacci sequence and extended Binet's formula, Integer 9 (2009), 639-654.

[3] S. Falcon and A. Plaza, On the Fibonacci $k$-numbers, Chaos, Solitons and Fractals 32 (2007), 1615-1624.

[4] V. K. Gupta, Y. K. Panwar and O. Sikhwal, Generalized Fibonacci Sequences, Theoretical Mathematics and Applications 2 (2) (2012), 115-124.

[5] R. J. Hendel, Approaches to the formula for nth Fibonacci number, College Math. J. 25 (1994), 139-142.

[6] S. Ikikardes and Z. Sarigedik, Some properties of the generalized Fibonacci and Lucas sequences related to the extended Hecke groups, Journal of Inequalities and Applications 2013, 2013:398.

[7] D. Kalman and R. Mena, The Fibonacci numbers - Exposed, The Mathematical Magazine 2 (2002)

[8] E. Kilic, Sums of the squares of terms of sequence $\left\{u_{n}\right\}$, Proc. Indian Acad. Sci.(Math. Sci.) Vol. 118, No. 1, February 2008, 27-41.

[9] I. Niven and H. S. Zuckerman, An introduction to the Theory of Numbers, 2nd ed., Wiley, New York, 1966.

[10] J. R. Silvester, Fibonacci properties by matrix methods, Mathematical Gazette 63 (1979), 188-191.

[11] B. Singh, O. Sikhwal and S. Bhatnagar, Fibonacci-Like sequence and its properties, Int. J. Contemp. Math. Sciences 5 (18) (2010), 895-868.

[12] D. Vella and A. Vella, Cycles in the Generalized Fibonacci Sequence modulo a Prime, The Mathematical Magazine 75 (4) (2002), 294-299.

Young Ho Jang

Department of Mathematics

Inha University

Incheon 402-751, Korea

E-mail: yjang6105@inha.ac.kr

Sang Pyo Jun

Information Communication

Namseoul University

Cheonan 331-707, Korea

E-mail: spjun7129@naver.com 